\title{
Segmentation of the proximal femur in radial MR scans using a random forest classifier and deformable model registration
}

Dimitrios Damopoulos ${ }^{1}$, Till Dominic Lerch ${ }^{2}$, Florian Schmaranzer ${ }^{2}$, Moritz Tannast ${ }^{2}$, Christophe

Chênes ${ }^{3}$, Guoyan Zheng ${ }^{1, *}$, Jérôme Schmid ${ }^{3, *}$

${ }^{1}$ Institute for Surgical Technology and Biomechanics, University of Bern,

Stauffacherstrasse 78, 3014 Bern, Switzerland

${ }^{2}$ Department of Orthopaedic Surgery and Traumatology, Inselspital, University of Bern, Freiburgstrasse, 3010 Bern, Switzerland

${ }^{3}$ School of Health Sciences - Geneva, HES-SO University of Applied Sciences and Arts Western Switzerland, Avenue de Champel 47, 1206 Geneva, Switzerland

* Guoyan Zheng and Jérôme Schmid share the senior authorship

\section{Corresponding Authors}

Dimitrios Damopoulos

Email: dimitrios.damopoulos@istb.unibe.ch

Office telephone number: +41316315949

Guoyan Zheng

Email: guoyan.zheng@istb.unibe.ch

Office telephone number: +41316315956 


\section{Abstract}

\section{Background}

Radial 2D MRI scans of the hip are routinely used for the diagnosis of the camtype of femoroacetabular impingement (FAI) and of avascular necrosis (AVN) of the femoral head, both considered causes of hip joint osteoarthritis in young and active patients. A method for automated and accurate segmentation of the proximal femur from radial MRI scans could be very useful in both clinical routine and biomechanical studies. However, to our knowledge, no such method has been published before.

\section{Purpose}

The aims of this study are the development of a system for the segmentation of the proximal femur from radial MRI scans and the reconstruction of its $3 \mathrm{D}$ model that can be used for diagnosis and planning of hip-preserving surgery.

\section{Methods}

The proposed system relies on: (a) a random forest classifier and (b) the registration of a $3 \mathrm{D}$ template mesh of the femur to the radial slices based on a physicallybased deformable model. The input to the system are the radial slices and the manually-specified positions of three landmarks. Our dataset consists of the radial MRI scans of 25 patients symptomatic of FAI or AVN and accompanying manual segmentation of the femur, treated as the ground truth.

\section{Results}

The achieved segmentation of the proximal femur has an average Dice similarity coefficient (DSC) of $96.37 \pm 1.55 \%$, an average symmetric mean absolute distance (SMAD) of $0.94 \pm 0.39 \mathrm{~mm}$ and an average Hausdorff distance of $2.37 \pm 1.14 \mathrm{~mm}$. In the femoral head sub-region, the average SMAD is $0.64 \pm 0.18 \mathrm{~mm}$ and the average Hausdorff distance is $1.41 \pm 0.56 \mathrm{~mm}$.

\section{Conclusions}

We validated a semi-automated method for the segmentation of the proximal femur from radial MR scans. A 3D model of the proximal femur is also reconstructed, which can be used for the planning of hip-preserving surgery.

\section{Keywords}

radial imaging of the hip; proximal femur; 3D reconstruction; segmentation; random forest; deformable model 


\section{Introduction}

Femoroacetabular Impingement (FAI) and avascular necrosis of the femoral head (AVN) are known causes of osteoarthritis of the hip joint in young and active patients [1]. FAI has been described in [2] as a biomechanical entity that originates from anatomical abnormalities of the proximal femur and/or the acetabulum which manifests itself with decreased range of motion and pain [3-7]. FAI is the main cause of early-onset osteoarthritis in non-dysplastic hips [4]. In the cam-type of FAI, anatomical abnormalities are observed only on the femoral head without any pelvis involvement. On the other hand, in AVN the blood flow to the femoral head is interrupted, which can progressively lead to the collapse of the hip. A lot of jointpreserving forms of treatment have been developed in an attempt to slow or reverse its progression, as it usually affects young patients $[1,8]$. MRI has been recognized as an important assisting tool for the diagnosis and the assessment of FAI and AVN as, in addition to the non-ionizing nature of its radiation, MRI can capture the vascular status of the femoral head [9-12]. Moreover, as MR scanners typically have the capability to directly scan planes of arbitrary orientation, it is possible to acquire images perpendicular to the curvature of the acetabulum, as visualized in Fig. 1. Such a scanning protocol is often referred to as radial imaging of the hip. The appeal of using radial scans over 3D MRI for image-assisted diagnosis is the reduced scanning time, as a typical radial scan of the hip consists of much fewer slices. Indeed, acquisition of 3D MR images is typically not part of the clinical routine, as MR scanning time tends to be a resource high in demand. Additionally, radial imaging, using a gadolinium-enhanced scanning protocol [13-16], is considered the gold standard for diagnosis of FAI and AVN $[1,17,18]$.

In addition to the value of MRI in diagnosis, MR-based 3D models of the femur have recently been shown to be as accurate as CT-based ones [19,20]. Such 3D models form an essential part of the planning of hip-preserving surgeries and they are also valuable in conducting motion analysis studies. In particular, surgical correction of cam-FAI or AVN is challenging and requires exact pre-operative planning [5-8]. The reconstruction of such a 3D model requires the segmentation of the femoral area in every slice, a task which, if performed manually, is very tedious. In the case of MR radial scans, the effort required is much lower. Still, the segmentation result is susceptible to inter-observer variability. Moreover, radial scans can suffer from a crosstalk artifact at the intersection of all the images and it can be unclear how to segment the proximal femur at the affected locations.

For these reasons, an automated and accurate method for the segmentation of the proximal femur in radial scans of the hip has the potential to increase the value of this type of sequence in both clinical routine and biomechanical studies. In this work, we present a novel method for this task. The proposed method registers a 3D template mesh of the femur to the radial slices based on a physically-based deformable model. The registration process utilizes the pixel-wise predictions of a classifier. An evaluation study is conducted on a dataset of 25 radial scans of symptomatic patients. 


\section{Related Work}

There are a few published methods on the problem of the automatic segmentation of the proximal femur from 3D MR images. In [21], the authors applied both multiatlases and active shape models (ASMs) for the segmentation of the proximal femur and the hip bone. In [22-25], different methods based on deformable models were proposed: In [22], a robust multi-resolution statistical shape model (SSM) method for the segmentation of bones in small field-of-view (FOV) was presented and results for the case of the femur and of the hip bone were reported. In [23], a general segmentation method for both muscles and bones was presented and an evaluation for femur segmentation was conducted. In [24], user-specified landmarks were also utilized for the segmentation of the proximal femur. In [25], weighted shape prior were introduced to the deformable model. Whereas the previous methods are all model-based, in [26] the authors presented a purely classification-based approach, by proposing a 3D deep learning network architecture based on u-net for this classification task.

All the methods mentioned above assume that 3D MR data are available. To the best of our knowledge, no segmentation method for the segmentation of the proximal femur has been proposed that relies solely on radial scans. Turning our attention to different organs, we can find literature on the segmentation of heart from similar radial images, for example [27,28]. As summarized in [27], these methods tend to have an interpolation part and a segmentation part. Sometimes, the slices are segmented independently from one another and the 3D surface is interpolated by the $2 \mathrm{D}$ contours. Alternatively, often a $3 \mathrm{D}$ volume is firstly interpolated from the $2 \mathrm{D}$ slices and then some segmentation method, usually model-based, is applied on the $3 \mathrm{D}$ volume.

The geometric feature that we are using in the classification stage is similar to those of the auto-context framework [29], especially to the distance-based features introduced in [30]. The main difference in these works is that they employ at least one prior regression or classification layer in order to locate certain landmarks or objects. In the present work, we attempt to directly take advantage of prior knowledge concerning the orientation of the images without a prior localization step. 


\section{Method}

The input to the pipeline is a set of radial MR images of the hip. Their field of view includes the hip joint and the upper extremity of the femur. In the following sections, we will be referring to such an MR sequence as a radial scan or simply $a$ scan. For the individual planes of a radial scan, we will use the term images.

Unlike typical MR scans, the images of a radial scan are not parallel to each other. Instead, they lay on oblique planes, acquired in a radial (rotating) fashion along the axis of the femoral neck. Their acquisition protocol is described in detail in [13], which specifies 14 images per scan. Following this protocol, the images are gadolinium-enhanced. The geometric arrangement is visualized in Fig. 1, which presents an example radial scan from different $3 \mathrm{D}$ viewpoints.

The proposed pipeline consists of: (a) a pre-processing step, where the input images are aligned; (b) a classification step with a random forest classifier that estimates the probability of every pixel to belong to femur; (c) a deformable model registration stage which fits a $3 \mathrm{D}$ template mesh to the input images and the generated probability maps. A summarizing diagram is presented in Fig. 2. Each component is described in the following sections.

\section{Preprocessing}

At the preprocessing stage, two operations are carried out: (a) the intensities of the images are normalized by histogram matching to a reference image and (b) the images are rotated so that the common axis is vertical on every aligned image.

The intensity normalization is performed because the range of the intensities of an image can be different for different radial scans, which might affect the performance of the intensity-based features that are used by the random forest classifier. The matching is performed using a standard algorithm for histogram matching for MR images [31].

In order to reduce the variability of the appearance of the femur in the images and to assist the classification stage that follows, the images are rotated so that the common axis is always vertical. This is accomplished in the following manner: Firstly, the common axis is retrieved by computing the intersection of any two images in the scan. Then, the orientation of the common axis with respect to every image is calculated. The desired rotation angle is the opposite of the angle of the computed orientation. The result of this alignment procedure is shown in Fig. 3.

The locations of three anatomical landmarks are also specified manually by the user, to be used for the initialization of the deformable model registration procedure of the last stage.

\section{Random forest classifier}

The automated segmentation is based on a binary, pixel-wise classifier which makes a probabilistic estimate for every pixel of an aligned image on whether it 
belongs to the femur area or not. These two classes of pixels will be referred to as foreground and background in the following sections.

A random forest is utilized for the binary classification. In addition to standard, intensity-based features, a geometric feature is used that attempts to introduce spatial context information to the classification process. For the description of these features that follows, it is assumed that an image $I: \Omega \subset \mathbb{R}^{2} \rightarrow \mathbb{R}$ is given, with the domain $\Omega$ corresponding to the pixel coordinates of the image. Since the classifier treats the images of a scan independently from each other, we ignore the fact that the pixels correspond in fact to $3 \mathrm{D}$ points in the world coordinate system and, for this sub-section, we treat the images as $2 \mathrm{D}$ objects.

\section{Intensity-based features}

A standard type of intensity-based features are utilized, the same way as in $[32,33,44-46,49]$. These features are based on the mean intensity value over displayed boxes. In more detail, if $\boldsymbol{x} \in \Omega$ is a reference point, the following features are considered:

$$
f\left(\boldsymbol{x} ; B_{1}, B_{2}, \boldsymbol{o}_{1}, \boldsymbol{o}_{2}, s\right)=\frac{\sum_{\boldsymbol{y} \in B_{1}} I\left(\boldsymbol{x}+\boldsymbol{o}_{1}+\boldsymbol{y}\right)}{\left|B_{1}\right|}-s \frac{\sum_{\boldsymbol{y} \in B_{2}} I\left(\boldsymbol{x}+\boldsymbol{o}_{2}+y\right)}{\left|B_{2}\right|}
$$

Where $s \in\{0,1\}, \boldsymbol{o}_{1}, \boldsymbol{o}_{2}$ are $2 \mathrm{D}$ offsets, $\boldsymbol{B}_{1}, \boldsymbol{B}_{2}$ are $2 \mathrm{D}$ rectangular boxes and $\left|B_{1}\right|,\left|B_{2}\right|$ are their areas.

As in the previously mentioned publications, a pool of these features is sampled randomly from a predefined range of values for $\boldsymbol{B}_{1}, \boldsymbol{B}_{2}, \boldsymbol{o}_{1}, \boldsymbol{o}_{2}$ at the beginning of the random forest training. The interested reader is referred to [46] for a detailed description of this family of features and how they are integrated in the random forest classification framework.

\section{A geometric feature}

In order to improve the performance, we take advantage of prior knowledge concerning the geometric arrangement of the images in a scan. As described earlier, the images of a radial scan of the proximal femur intersect on one axis in the $3 \mathrm{D}$ world coordinates space which passes close to the femoral head center and the femoral neck center. This prior knowledge is incorporating in the classifier through a feature which is simply the distance of the reference point to the common axis. This can be expressed as:

$$
g(\boldsymbol{x} ; \boldsymbol{s})=\left\|\left(\boldsymbol{x}-\boldsymbol{s}_{0}\right)-\left(\left(\boldsymbol{x}-\boldsymbol{s}_{0}\right)^{\boldsymbol{T}} \cdot \hat{\boldsymbol{s}}\right) \hat{\boldsymbol{s}}\right\|_{2}
$$

Where $\boldsymbol{s}_{0}$ is a point of the common axis and $\hat{\boldsymbol{s}}$ is the direction vector of the common axis. This is more intuitively illustrated in Fig. 3(e). As it can be seen on this figure, this distance is calculated on the aligned image, so the unit vector $\hat{\boldsymbol{s}}$ is simply 
equal to $[0,1]^{T}$ and the computation above simplifies to just the subtraction of the y coordinate of $\boldsymbol{s}$ from the y coordinate of $\boldsymbol{x}$.

\section{Deformable model fitting}

In the last stage, a 3D template mesh of the femur is registered to the radial images based on a physically-based deformable model. The implemented deformable model framework is partially based on our previous work of physically-based simplex meshes [22,34]. In this framework, mesh vertices are considered as lumped mass particles whose motion is driven by forces and which follows Newtonian dynamics. By carefully crafting the forces, the deformable model will reach an equilibrium position corresponding to the structure to segment.

The state of a particle $i$ at time $t$ is described by its position $P_{i}(t)$ and velocity $P_{i}^{\prime}(t)$. Following Newton's law of motion, the particle acceleration $P_{i}^{\prime \prime}(t)$ is related to the inverse of the particle mass, expressed by its inertia matrix $L_{i}$, and the sum of forces $F_{i}$ exerted: $P_{i}^{\prime \prime}(t)=L_{i} F_{i}(t)$. The particle motion is thus described by a differential equation system which can be linearized and solved according to an integration scheme. We chose the Implicit Euler scheme as described in [35]:

$$
\begin{aligned}
& P(t+d t)=P(t)+P^{\prime}(t+d t) d t \\
& P^{\prime}(t+d t)=P^{\prime}(t)+L^{T} H^{-1} Y \\
& H=L^{T}-L \frac{\partial F}{\partial P^{\prime}} L^{T} d t-L \frac{\partial F}{\partial P} L^{T} d t^{2} \\
& Y=L F(t) d t+L \frac{\partial F}{\partial P} P^{\prime}(t) d t^{2}
\end{aligned}
$$

This scheme remains very stable when using large integration time steps and can be efficiently implemented by using the conjugate gradient algorithm and by exploiting the sparsity of the system matrix $H$. A simulation step consists of: (a) computing forces and their derivatives and (b) updating the next particle state by solving the differential equation system. For a vertex at position $P_{i}$, a force $f_{i}$ is commonly modelled as the force of a Hookean spring which will attract the vertex towards a target vertex position $R_{i}$ :

$$
f_{i}=\propto_{i}\left(R_{i}-P_{i}\right)
$$

where $\propto_{i}$ is the weighting force coefficient.

A key difference with respect to our previous framework $[22,34]$ is that we do not rely anymore on simplex meshes. We use instead standard triangular meshes due to the maturity and availability of many geometrical modelling techniques for this category of meshes. As a result, we devised new approaches in the framework, as detailed in the following paragraphs. 


\section{Internal forces}

Internal forces regulate mesh deformation by enforcing smoothness and shape similarity. Shape similarity is enforced by local and global shape forces. Smoothness and local shape forces focuses on a local geometric description of a vertex with respects to its neighbors, while strong shape forces are based on statistical shape models [36] and affect the mesh globally.

\section{Smoothing and local shape forces}

Contrary to triangular meshes, simplex meshes are characterized by constant vertex connectivity, simplifying the computation of various local geometrical properties that were used in our previous framework.

Instead of relying on the dual transformation between simplex and triangular meshes, we choose in the present work to model the vertex local geometry with the Mean-Value Encoding (MVE) of [37]. In MVE, for each vertex at position $P_{i}$ a local plane $\Pi_{i}\left(n_{i}, d_{i}\right)$ is built based on the $m_{i}$ neighbor vertex positions $Q_{j}, j \in N_{i}$. The plane normal $n_{i}$ is computed as an area-weighted sum of the neighbor vertex normals, where the area $a_{i}$ of a vertex $i$ is computed from the areas $A_{i k}$ of the triangles sharing the vertex: $a_{i}=\sum A_{i k} / 3$. The plane position $d_{i}$ is expressed as an average distance from the origin:

$$
d_{i}=-\frac{1}{m_{i}} \sum_{j \in N_{i}} n_{i} \cdot Q_{j}
$$

Each vertex position is expressed in terms of tangential and normal components with respect to the local plane, by a series of MVE parameters. These parameters provide an efficient encoding of local geometry at each vertex, robust to degenerate situations such as nearly collinear neighbor vertices.

MVE parameters can be precomputed for the mesh at $t=0$, providing a "snapshot" of the mesh shape. Then, during mesh deformation, for each vertex position $P_{i}$ a decoding process computes the position $R_{i}$ that would be expected based on the MVE encoding. The local shape force uses this position $R_{i}$ as the target position to enforce local shape geometry.

The MVE local geometry can also be used to design a smoothing force preventing excessive local deformations such as spokes. The target point $R_{i}$ for vertex $i$ of the smoothing force is:

$$
R_{i}=\sum_{j \in N_{i}} a_{j}\left(Q_{j}+h_{j}\right) / \sum_{j \in N_{i}} a_{j}
$$

where $h_{j}$ denotes the height of the vertex $j$ in its local geometry, i.e. the normal component of position $P_{j}$ with respect to its local plane $\Pi_{j}$. This smoothing is similar to Laplacian smoothing which uses the vertex barycenter but it additionally reduces the shrinking effect of the Laplacian smoothing by including the weighted height. 


\section{Global shape force}

In [22], we presented the use of statistical shape models (SSM), expressed as point distribution models [36], to create the global shape force. The idea is to compute a shape $S$ based on the SSM which is the closest in least squares sense to the current mesh $M$. This is based on an iterative procedure described in [36] which estimates the best alignment transform and shape parameters of the SSM yielding the closest shape $S$. The vertices of $S$ are eventually used as the target vertices $R_{i}$ for the global shape forces.

\section{External forces}

External image forces use image cues to guide the deformation of the mesh and are specifically designed based on the modality and nature of the images. In this work, we use both pixel intensities of the MR images and the values of the probability maps of the random forest classifier. Without any loss of generality, we will refer to any of them as image with intensities $I$.

Given the 3D plane of a radial image, we select the mesh vertices $P_{i}^{*}$ whose projection on the plane $L_{i}^{*}$ are within the image bounds and whose distance to the plane are below a very small threshold $l$ (Fig. 5(a)). Then, the normals of the vertices $P_{i}^{*}$ are projected on the plane as $2 \mathrm{D}$ vectors $n_{i}^{*}$. In the plane, we sample $2 W+1$ values every $s \mathrm{~mm}$ along the normal direction $n_{i}^{*}$ in an interval centered on $L_{i}^{*}$. The sampling position $u$ where the intensity gradient $\nabla I(u)$ has $(i)$ the greatest magnitude but also (ii) the closest direction to the projected normal $n_{i}^{*}$, is chosen for the force target point $R_{i}$ (Fig. 5(b)):

$$
R_{i}=\operatorname{argmax} \varepsilon * \nabla I(u) \cdot n_{i}^{*}
$$

where $\varepsilon$ equals +1 or -1 depending on whether the normals need inversion (in the case of probability maps, outward mesh normals and image gradients will have opposite directions). This force will only affect a subset of mesh vertices $P_{i}^{*}$ due to the small spatial coverage of the radial images. The remaining vertices are ignored during the alignment procedure and the least square minimization of the global shape force computation [22].

\section{Regularization}

Internal forces play an important role in preventing incorrect mesh deformations due to image forces affected by image noise or neighbor anatomical structures. However, additional regularization strategies are generally required to bring robustness and tackle possible numerical issues.

First, we adopt the same multi-resolution strategy depicted in [22], where several resolutions of the mesh are successively used during the mesh deformation. We also use a coarse-to-fine SSM scheme, by progressively increasing the SSM "locality" [34] during the process. The locality is related to the alignment type of the SSM and 
it is an intuitive notion: a rigid SSM captures global shape changes while an affine or a similarity SSM will better express local variations.

Second, we used a damping force $f_{i}=-\propto_{i} P_{i}^{\prime}$ to prevent possible instabilities of the integration scheme. This may occur during the approximation of the force derivatives or when particle interactions are ignored. Approximate derivative may be chosen to preserve the system symmetry required by the conjugate gradient method or to simplify computations. For instance, the global shape force computation for a vertex $i$ will actually depend on all particle positions at the previous time step. However, we intentionally (wrongly) assume that the resulting $R_{i}$ will not depend on the particle positions. In practice, neither instabilities nor odd behaviors were noticed with the chosen approximations. 


\section{Experimental Design and Results}

\section{Dataset}

Our dataset consists of 25 MR gadolinium-enhanced radial scans of 25 patients symptomatic of FAI or AVN. They were acquired in the period 2010-2016 in the Sonnenhofspital, Bern, Switzerland following a radial sequencing acquisition protocol of the hip joint [13]. The age of the patients is $16-47$ years with a mean age of 29.0 years, 11 are male and 14 are female. In 12 patients it is the left hip that is scanned and in 13 cases it is the right hip. The $3 \mathrm{D}$ geometry of an example radial scan is visualized in Fig. 1. The intra-slice spacing is in $0.28-0.29 \mathrm{~mm}$ range, the size of the images is either $448 \times 448$ ( 19 cases) or $512 \times 512$ ( 6 cases) and there are 14 images in every scan. Radial scans of this type are routinely acquired in the aforementioned hospital. A reference, manual segmentation of every image of the radial scans is also provided.

\section{Study design}

A 5-fold cross-validation study is performed on the dataset of the 25 radial scans with every fold consisting of five scans. Therefore, each scan is utilized exactly once as part of a test set. The reference image of the intensity normalization preprocessing step is set to be the image of the training set with the median mean intensity. For the initialization of the deformable model registration stage, the following three landmarks were used: (a) the femoral head center, (b) the femoral neck center and (c) the tip of the minor trochanter. These were specified manually for each case using the "Fiducials" module of the Slicer 3D open-source software [41].

\section{Hyper-parameters}

\section{Random forest}

Given the relatively small size of our dataset, the most preferable way to tune the hyper-parameters of the random-forest would be independently for every cross-validation iteration through a nested cross-validation scheme. However, due to the long training times (more than half a day for one iteration), we opted to set the hyperparameters in advance to fixed values, based on related literature and prior knowledge on the task at hand. Specifically, when random forests are utilized as classifiers for image segmentation problems (as in [42-46]), the maximum depth of the trees is usually in the $T \in[20,40]$ range and their number in the $N \in[20,120]$ range. These parameters affect the generability of the trained classifier: For a fixed depth, more trees reduce the variance of the model (but lead to higher training and testing times) and, for a fixed number of trees, the depth influences the bias-variance trade-off. These effects of $T, N$ can be explained theoretically [50] and they have been observed in practice [46-48]. We conservatively set the maximum depth to $T=20$ to prevent overfitting and the number of trees to $N=50$. The box-size of 
the intensity-based features is between $0.3 \mathrm{~mm}$, which is slightly larger than the pixel spacing in the dataset, and up to $7.5 \mathrm{~mm}$, as we expect that no area in the foreground larger than $7.5 \times 7.5 \mathrm{~mm}^{2}$ has uniform intensity. Following the practice of our prior work [49], we set the maximum range of the intensity features to double the maximum length of the boxes, i.e. to $15 \mathrm{~mm}$.

A posteriori, we varied the tree depth as $T \in\{15,20,25,30\}$, keeping the number of trees to $N=50$. We found out that for $\mathrm{T}=25$ for the classifier-based method, the mean scan DSC increased by $0.07 \%$ and the mean SMAD decreased by $0.02 \%$, suggesting that there is some room for better tuning of our method, probably though only marginal. Since we did not follow a nested cross-validation strategy, we are reporting here the results obtained with the preset parameter values.

\section{Deformable model}

The hyper-parameters of the deformable model registration stage were chosen based on an empirical analysis performed on three randomly chosen cases. The procedure was based on a semi-automatic interactive segmentation, during which a skilled operator could increase or decrease the influence of forces as well as increasing the resolution of the mesh when needed. Some initial parameters of the segmentation, such as weight forces or image force coverage, were derived from our previous experience in MRI segmentation of the hip [22]. From this previous work, we could already identify some best strategies to apply, such as the choice of the SSM alignment with respect to the mesh resolution. All operator actions were recorded in a script which could be reapplied later on in an automatic fashion on the test cases. If some high errors were detected, the operator would re-run the interactive segmentation and adapt her/his choices until a satisfactory segmentation was observed for the three cases. As a measure against over-fitting with respect to these cases, the script was cleaned out to keep the parameters as constant as possible, since the operator varied some of them unnecessarily over time. The parameters whose variation was found to have a significant impact on the results were the global shape force weight and image force coverage; only these two were allowed to evolve over time.

Using the strategy described above, the deformable model used 4 mesh resolutions, ranging from 700 to $50 \mathrm{~K}$ points. The mass of a vertex was set as the total surface of the mesh divided by the number of vertices. Mesh resolution 3 (the coarsest) used a rigidly aligned SSM, while resolution 2 and 1 used an SSM with affine alignment. The highest resolution 0 did not use any global shape force. The weighting force coefficients $\propto_{i}$ of the smoothing and local shape forces were respectively set to 0.2 and 0.3 . The global shape force weight progressively decreased from 0.8 to 0.1 during the simulation as follows: for $51 \%$ of the total iterations count the weight was set to 0.8 , then to 0.4 for $8 \%, 0.3$ for $13 \%, 0.1$ for $20 \%$ and 0 (the force being not used for the highest mesh resolution) during the remaining $8 \%$. The statistical shape models were built from training femur shapes produced by segmenting a collection of 200 hip CT images. The image force weight was kept constant at 0.05 for the MR image and 0.45 for the probability map, but the image force coverage decreases over time from $9(\mathrm{~W}=4)$ samples to $5(\mathrm{~W}=2)$ samples spaced by 
$s=1.2 \mathrm{~mm}$. The distance threshold 1 of the image force was set to $0.001 \mathrm{~mm}$. Finally, damping weight was set to 0.5 and the time step was 1.0.

\section{Evaluation metrics}

The accompanying manual segmentation of the proximal femur is treated as the ground truth in our study. We compare the generated segmentation with this reference segmentation with respect to area overlap and border distance.

The area overlap is quantified with the Dice similarity coefficient (DSC) and two types of DSC measurements are performed: (a) the DSC is computed independently for every $2 \mathrm{D}$ images and (b) all the images of a scan are treated as a single set and their DSC is computed with respect to the set of the image segmentations.

The two borders are compared using the symmetric mean absolute distance (SMAD) measure and the Hausdorff distance measure. The segmentation of every radial scan is treated as a single set, thus all the images of a scan are taken into consideration. We also repeat these measurements specifically for the femoral head area by isolating the region from the hip joint to the femoral head center using a 3D mask.

The achieved scores on these metrics are presented on Table 1. In Fig. 6, the images of the radial scan with the median scan DSC value $(96.65 \%)$ are listed, overlaid with the reference segmentation and the output of our method. Subfigures (e) and (f) of Fig. 1 illustrate an example registered 3D model of a testing case, along with two of its radial images.

When the three cases that were utilized for the configuration of the deformable model stage are omitted from the evaluation, the mean SMAD gets slightly better $(0.62 \mathrm{~mm}$.instead of 0.64$)$, the scan DSC gets slightly worse (96.34 instead of 96.40 $\%)$ and the mean values of the remaining 4 evaluation metrics stay the same, suggesting that no significant bias was introduced by the inclusion of these three cases in the evaluation.

\section{Evaluation of specific components of the pipeline}

The study was repeated for two variants of the proposed method, using the same five folds, in order to quantify the effect of specific components of the pipeline.

Firstly, we assess the effect of the deformable model registration stage on the segmentation performance by comparing the proposed pipeline with an alternative one that is based on the classifier without any registration of a 3D model. In order to make the comparison fair, we improve the result of the alternative method with standard post-processing operations: Firstly, a Conditional Random Field (CRF) [38] with a simple Potts model is applied, whose unary potentials are the probabilistic prediction of the random forest. The CRF inference is performed using the DGM C++ library [51]. Then, any holes on the resulting segmentation are filled and its largest connected component is the final output. The performance of this classifier-based method is summarized on Table 2. 
Secondly, the importance of the geometric feature is assessed by measuring the segmentation performance when this feature is not utilized. The scores achieved are presented on Table 3.

Statistical tests were conducted for each of the two variants in order to determine whether their performance differs significantly from the default pipeline, in the sense that is described in this paragraph. In particular, for each of the six evaluation metrics, a two-sided Wilcoxon signed-rank test was performed, hereinafter referred to as the WSR test. Let $m_{i}, v_{i}, i \in\{1, \cdots, N\}$ be the measurements of two methods $M, V$, as obtained on a set of $N$ samples. Let also $D_{M}, D_{V}$ denote the distributions that $m_{i}, v_{i}$ respectively are sampled from. The WSR test examines the differences $x_{i}=m_{i}-v_{i}$, whose distribution is denoted with $D_{X}$. The test makes the following assumptions: that the $N$ samples are chosen randomly, that the values of the metric can be treated as continuous (so that expressions like $m_{i}-v_{i}$ are meaningful) and that $D_{X}$ is approximately symmetric around its median value $\theta_{X}$. Its null hypothesis is the following [52-54]:

Null hypothesis of the WSR test: $\theta_{X}=0$.

The WSR test was chosen over the paired samples t-test, whose null hypothesis is that the mean value of $D_{X}$ is zero, because the distributions of the values were found be to non-Gaussian. As a counteraction to the well-known Multiple comparisons problem, after the conduction of the six tests, the Holm-Bonferroni correction method was applied. The significance level for all the statistical tests was set to 0.05 . The resulting (corrected) p-values are reported in the rightmost columns of Table 2 and Table 3. 


\section{Discussion and conclusion}

\section{Performance of the proposed pipeline}

We performed a validation study on the radial MR scans of 25 patients symptomatic of FAI or AVN. The proposed pipeline yields satisfactory results in terms of both area overlap and border distance to the reference segmentation. As summarized on Table 1, the mean DSC of the segmentation is $96.37 \%$, the mean SMAD is 0.94 $\mathrm{mm}$ and the mean Hausdorff distance is $2.37 \mathrm{~mm}$. There is generally no significant difference between computing the DSC of the whole scan and computing the mean DSC of its images, as the femur occupies more or less the same area in all the images.

On Fig. 6 it can be qualitatively observed that the largest difference with the manual segmentation of a typical case (the one with the median DSC) occurs near the trochanters, whereas there is less difference near the joint space. This agrees with the results of Table 1, as the average SMAD and the average Hausdorff distance is much lower when calculated only on the femoral head $(0.64 \mathrm{~mm}$ and 1.41 $\mathrm{mm}$ respectively, compared to $0.94 \mathrm{~mm}$ and $2.37 \mathrm{~mm}$ ). We think that this is important for certain applications, since frequently the most critical part of the proximal femur is the one close to the hip joint. It also demonstrates the ability of the proposed method to follow the border of the proximal femur in the regions affected by cross-talk artifact of the radial scans, which can be challenging during manual segmentation.

Some representative images from six scans are shown on Fig. 7, each exemplifying typical pathological findings and/or challenges for the automated segmentation (three cases where the femoral heads have a highly non-spherical shape and three cases with cam lesions). The border of the automated segmentation is also shown. The pipeline copes with these variations in most cases satisfactory, but mistakes do happen. In Fig. 7, it can be observed that the method did not manage to follow the border of a non-spherical femoral head in a sub-region of one case and that a small cam-type lesion was segmented-out in another image.

In terms of runtime performance, the proposed pipeline is relatively fast: On a system with a standard Intel i7 CPU dual-core at $2.7 \mathrm{GHz}$, the random forest classification takes about 2.5 seconds per slice, therefore 35 seconds for the 14 images of a scan. The subsequent deformable model registration takes approximatively 40 seconds. The pre-processing stage completes in around 10 seconds, thus the pipeline needs around 1.5 minute in total to segment the 14 images of a scan and to reconstruct the 3D model of the proximal femur.

\section{Effect of the geometric feature}

When only intensity-based features are used, the probability maps frequently have high values for areas which are far away from the femur and the border be- 
tween background and foreground is not always sharp. These problems are illustrated on Fig. 4(b). After the inclusion of the geometric feature, the situation is much improved (Fig. 4(c)). The effect in performance is quantified in Table 3, which presents the performance of a pipeline that uses only intensity-based features in the classification process: The average, standard deviation, median, minimum and maximum values of all the evaluation metrics get worse, with the average scan DSC decreasing from $96.37 \%$ to $95.80 \%$; the average SMAD increasing from $0.94 \mathrm{~mm}$ to $1.08 \mathrm{~mm}$ and the average Hausdorff distance increasing from $2.37 \mathrm{~mm}$ to 2.59 $\mathrm{mm}$.

This variant was compared with the default pipeline using a WSR test for each evaluation metric, followed by a correction of the p-values with the Holm-Bonferroni method. Within the confidence level of $95 \%$, the difference with the default pipeline with respect to the Hausdorff distance metric was found to not be statistically significant. The difference with respect to each of the remaining five metrics was found to be statistically significant within the confidence level of $95 \%$.

\section{Importance of deformable model registration stage}

We also compared the proposed system with a purely classifier-based approach. We found that the latter yields slightly worse mean values of all the six evaluation metrics (Table 2): The average scan DSC decreases from $96.37 \%$ to $95.69 \%$; the average SMAD increases from $0.94 \mathrm{~mm}$ to $1.12 \mathrm{~mm}$ and the average Hausdorff distance increases from $2.37 \mathrm{~mm}$ to $2.75 \mathrm{~mm}$. The performance of the classifierbased variant was compared with that of the default pipeline using a WSR test for each evaluation metric, followed by a correction of the p-values with the HolmBonferroni method. Within the confidence level of $95 \%$, the difference with the default pipeline with respect to each metric was found to be statistically significant.

In Fig. 8, the difference in performance between the two variants can be assessed visually. This figure presents box-and-whisker plots for the two variants for all the six evaluation metrics. A first observation that we can make from these plots is that there are strong outliers for all the metrics, indicating that their distributions cannot be considered Gaussian. Secondly, the boxes of the plots illustrate the fact that both the median values of the metrics and their distance from the lower quartile $\left(\mathrm{Q}_{1}\right)$ to the higher quartile $\left(\mathrm{Q}_{3}\right)$, i.e. their interquartile ranges $(\mathrm{IQR})$, are generally better with the deformable model (the only exception is the IQR for the Hausdorff distance, which does not change significantly). Visually, it seems that the difference between the two variants is least significant with respect to the Hausdorff distance in the femur head area and most significant with respect to the SMAD in the femur head area. Both observations agree with the minimum and maximum computed pvalues that are reported in Table 2.

We observed that the biggest performance gains with the deformable model registration frequently occur on the scans which are the most challenging to the classifier. For example, the classifier-based method performed worst in the cases with Ids 4,9 and 12, with average scan DSCs of $90.95 \%, 89.58 \%$ and $93.53 \%$ and average 
SMADs of $2.21 \mathrm{~mm}, 3.04$ and 1.60 respectively. The hip joint of case 4 has image findings consistent with Perthes disease and the cases 9 and 12 correspond to the youngest patients of our dataset (16 years old). Within the variance observed in our dataset, all these three proximal femurs have non-typical appearance. In these cases, the proposed pipeline achieves scan DSCs $90.90 \%, 95.34 \%$ and $95.44 \%$ and average SMADs $2.20 \mathrm{~mm}, 1.39 \mathrm{~mm}$ and $1.12 \mathrm{~mm}$ for case 4, 9 and 12 respectively. Our interpretation of these results is that, in some scans, the proximal femur has an appearance that has not been encountered during training and, hence, the classifier has difficulty in capturing its borders accurately. When this happens, the registration of the 3D mesh can help by taking advantage of its explicit modeling of the global shape of the femur.

We can summarize that the advantage of the purely classifier-based approach is that it performs only slightly worse than the complete pipeline while it remains fully automated, as it does not need to be initialized using manually-specified landmarks. The full pipeline proceeds with the registration of a deformable model of the proximal femur, which we found to improve the segmentation performance. While it is true that this improvement in performance is small, the resulting registered model of the proximal femur has important implications for clinical applications. Indeed, the output of a classifier is only a binary segmentation mask, whereas the registered model provides rich contextual interpretation of the segmentation result. The latter can be used for the direct localization of anatomical landmarks and for further planning of surgical planning procedures. For example, it permits the direct computation of clinically-relevant morphometric features, such as the head and neck diameters, the sphericity of the head, the length of the proximal femur and the intertrochanteric distance.

\section{Conclusion}

We present a pipeline for the segmentation of the proximal femur from radial scans of the hip and the reconstruction of its $3 \mathrm{D}$ model. We performed a 5 -fold cross validation on a dataset of 25 radial scans of patients symptomatic of FAI or AVN. With respect to a manual, reference segmentation of the proximal femur, the resulting segmentation has an average DSC of $96.37 \%$, an average mean SMAD of 0.94 $\mathrm{mm}$ and an average Hausdorff distance of $2.37 \mathrm{~mm}$. In the femoral head sub-region, the average SMAD is $0.64 \mathrm{~mm}$ and the average Hausdorff distance is $1.41 \mathrm{~mm}$.

In our view, the main limitation of the presented study is the lack of a comparison with the segmentation achieved from 3D MRI scans. Such an extension of our study is significant, as it will permit the detailed evaluation of the fitted 3D model and the examination of how the segmentation accuracy is affected by the lack of the dense intensity information of the 3D MRI. Unfortunately, we did not have accompanying 3D CT or MRI scans for the cases of our dataset, so such a study was not possible. In the future, we plan to collect additional 3D images, permitting us to further investigate the potential of a method based solely on radial scans to provide a reliable 3D model of the proximal femur. 
As part of future work, we also intend to replace the manual picking of the three landmarks for the initialization of the deformable model registration stage with an automated method, thus rendering the whole pipeline fully automated. For this purpose, we could employ a random forest regression localization approach, akin to our previous work [39]. Another possible extension important from a clinical standpoint is the concurrent segmentation of the acetabulum: the critical attribute of FAI is a decreased range of motion, therefore the modeling of the whole hip joint is a necessary component of a complete system what aims to facilitate the management of the condition. As the acetabulum socket is within the field-of-view of the radial scans, we expect that the classification stage can be directly extended for this task by simply adding a third class corresponding to the acetabulum. The registration of a 3D model of the acetabulum could be performed with the currently employed method, with the necessary inclusion of a collision-detection step that considers both parts of the hip joint.

In conclusion, surgical correction of cam-FAI or AVN is challenging and requires exact preoperative planning. The 3D model of the proximal femur as reconstructed by the proposed method can be utilized for this planning, as it does not require any modification to the MR protocol for these frequent hip diseases. We intend to use this method for diagnosis and planning of surgical correction of camFAI or AVN because, unlike 3D MRI, radial MR scans can be included in a routine MRI of the hip. 


\section{Funding}

This study was funded by the Swiss National Science Foundation (Grant number 205321_163224).

\section{Compliance with ethical standards}

All procedures performed in studies involving human participants were in accordance with the ethical standards of the institutional and/or national research committee and with the 1964 Helsinki declaration and its later amendments or comparable ethical standards.

Conflict of Interest: The authors Dimitrios Damopoulos, Till Dominic Lerch, Florian Schmaranzer, Moritz Tannast, Christophe Chênes, Guoyan Zheng and Jérôme Schmid declare that they have no conflict of interest.

Informed consent: Informed consent was obtained from all individuals included in the study. 


\section{References}

[1] Chughtai, M., Piuzzi, N.S., Khlopas, A., Jones, L.C., Goodman, S.B. and Mont, M.A., 2017. An evidence-based guide to the treatment of osteonecrosis of the femoral head. Bone Joint J, 99(10), pp.1267-1279.

[2] Sullivan, J.P., Griffith, T.B., Park, C.N. and Ranawat, A.S., 2017. Advances in 2D and 3D Imaging for FAI Surgical Planning. In Hip Joint Restoration (pp. 277285). Springer, New York, NY.

[3] Leunig, M., Beaulé, P.E. and Ganz, R., 2009. The concept of femoroacetabular impingement: current status and future perspectives. Clinical orthopaedics and related research, 467(3), pp.616-622.

[4] Tannast, M., Siebenrock, K.A. and Anderson, S.E., 2007. Femoroacetabular impingement: radiographic diagnosis - what the radiologist should know. American Journal of Roentgenology, 188(6), pp.1540-1552.

[5] Steppacher, S.D., Huemmer, C., Schwab, J.M., Tannast, M. and Siebenrock, K.A., 2014. Surgical hip dislocation for treatment of femoroacetabular impingement: factors predicting 5-year survivorship. Clinical Orthopaedics and Related Re$\operatorname{search} \AA, 472(1)$, pp.337-348.

[6] Steppacher, S.D., Lerch, T.D., Gharanizadeh, K., Liechti, E.F., Werlen, S.F., Puls, M., Tannast, M. and Siebenrock, K.A., 2014. Size and shape of the lunate surface in different types of pincer impingement: theoretical implications for surgical therapy. Osteoarthritis and cartilage, 22(7), pp.951-958.

[7] Lerch, T.D., Todorski, I.A., Steppacher, S.D., Schmaranzer, F., Werlen, S.F., Siebenrock, K.A. and Tannast, M., 2018. Prevalence of Femoral and Acetabular Version Abnormalities in Patients With Symptomatic Hip Disease: A Controlled Study of 538 Hips. The American journal of sports medicine, p.0363546517726983.

[8] Morita, D., Hasegawa, Y., Okura, T., Osawa, Y. and Ishiguro, N., 2017. Long-term outcomes of transtrochanteric rotational osteotomy for non-traumatic osteonecrosis of the femoral head. Bone Joint J, 99(2), pp.175-183.

[9] Petchprapa, C.N., Dunham, K.S., Lattanzi, R. and Recht, M.P., 2013. Demystifying radial imaging of the hip. Radiographics, 33(3), pp.E97-E112.

[10] Chana, R., Noorani, A., Ashwood, N., Chatterji, U., Healy, J. and Baird, P., 2006. The role of MRI in the diagnosis of proximal femoral fractures in the elderly. Injury, 37(2), pp.185-189.

[11] Cabarrus, M.C., Ambekar, A., Lu, Y. and Link, T.M., 2008. MRI and CT of insufficiency fractures of the pelvis and the proximal femur. American journal of roentgenology, 191(4), pp.995-1001.

[12] Sutter, R., Dietrich, T.J., Zingg, P.O. and Pfirrmann, C.W., 2012. How useful is the alpha angle for discriminating between symptomatic patients with camtype femoroacetabular impingement and asymptomatic volunteers?. Radiology, 264(2), pp.514-521.

[13] Klenke, F.M., Hoffmann, D.B., Cross, B.J. and Siebenrock, K.A., 2015. Validation of a standardized mapping system of the hip joint for radial MRA sequencing. Skeletal radiology, 44(3), pp.339-343. 
[14] Domayer, S.E., Mamisch, T.C., Kress, I., Chan, J. and Kim, Y.J., 2010. Radial dGEMRIC in developmental dysplasia of the hip and in femoroacetabular impingement: preliminary results. Osteoarthritis and cartilage, 18(11), pp.1421-1428.

[15] Zilkens, C., Tiderius, C.J., Krauspe, R. and Bittersohl, B., 2015. Current knowledge and importance of dGEMRIC techniques in diagnosis of hip joint diseases. Skeletal radiology, 44(8), pp.1073-1083.

[16] Riley, G.M., McWalter, E.J., Stevens, K.J., Safran, M.R., Lattanzi, R. and Gold, G.E., 2015. MRI of the hip for the evaluation of femoroacetabular impingement; past, present, and future. Journal of Magnetic Resonance Imaging, 41(3), pp.558-572.

[17] Schmaranzer, F., Todorski, I.A.S., Lerch, T.D., Schwab, J., Cullmann-Bastian, J. and Tannast, M., 2017, November. Intra-articular Lesions: Imaging and Surgical Correlation. In Seminars in musculoskeletal radiology (Vol. 21, No. 05, pp. 487-506). Thieme Medical Publishers.

[18] Schmaranzer, F., Haefeli, P.C., Hanke, M.S., Liechti, E.F., Werlen, S.F., Siebenrock, K.A. and Tannast, M., 2017. How does the dGEMRIC index change after surgical treatment for FAI? A prospective controlled study: preliminary results. Clinical Orthopaedics and Related Research $\AA$, 475(4), pp.1080-1099.

[19] Rathnayaka, K., Momot, K.I., Noser, H., Volp, A., Schuetz, M.A., Sahama, T. and Schmutz, B., 2012. Quantification of the accuracy of MRI generated 3D models of long bones compared to CT generated 3D models. Medical Engineering and Physics, 34(3), pp.357-363.

[20] Lerch T., Degonda C., Zheng G., Todorski I., Schmaranzer F., Ecker T., Siebenrock K. and Tannast M., 2017, MR-based 3D PAO planning and simulation of hip impingement is as accurate as CT-based 3D models, German Congress of Orthopedic and Trauma Surgery (DKOU 2017).

[21] Xia, Y., Fripp, J., Chandra, S.S., Schwarz, R., Engstrom, C. and Crozier, S., 2013. Automated bone segmentation from large field of view 3D MR images of the hip joint. Physics in Medicine \& Biology, 58(20), p.7375.

[22] Schmid, J., Kim, J. and Magnenat-Thalmann, N., 2011. Robust statistical shape models for MRI bone segmentation in presence of small field of view. Medical Image Analysis, 15(1), pp.155-168.

[23] Gilles, B. and Magnenat-Thalmann, N., 2010. Musculoskeletal MRI segmentation using multi-resolution simplex meshes with medial representations. Medical Image Analysis, 14(3), pp.291-302.

[24] Arezoomand, S., Lee, W.S., Rakhra, K.S. and Beaulé, P.E., 2015. A 3D active model framework for segmentation of proximal femur in MR images. International journal of computer assisted radiology and surgery, 10(1), pp.55-66.

[25] Chandra, S.S., Xia, Y., Engstrom, C., Crozier, S., Schwarz, R. and Fripp, J., 2014. Focused shape models for hip joint segmentation in 3D magnetic resonance images. Medical Image Analysis, 18(3), pp.567-578.

[26] Zeng, G., Yang, X., Li, J., Yu, L., Heng, P.A. and Zheng, G., 2017, September. 3D U-net with Multi-level Deep Supervision: Fully Automatic Segmentation 
of Proximal Femur in 3D MR Images. In International Workshop on Machine Learning in Medical Imaging (pp. 274-282). Springer, Cham.

[27] Paiement, A., Mirmehdi, M., Xie, X. and Hamilton, M.C., 2014. Integrated segmentation and interpolation of sparse data. IEEE Transactions on Image Processing, 23(1), pp.110-125.

[28] Van Assen, H.C., Danilouchkine, M.G., Frangi, A.F., Ordás, S., Westenberg, J.J., Reiber, J.H. and Lelieveldt, B.P., 2006. SPASM: a 3D-ASM for segmentation of sparse and arbitrarily oriented cardiac MRI data. Medical Image Analysis, 10(2), pp.286-303.

[29] Tu, Z., 2008, June. Auto-context and its application to high-level vision tasks. In Computer Vision and Pattern Recognition, 2008. CVPR 2008. IEEE Conference on (pp. 1-8). IEEE.

[30] Gao, Y., Wang, L., Shao, Y. and Shen, D., 2014, September. Learning distance transform for boundary detection and deformable segmentation in ct prostate images. In International Workshop on Machine Learning in Medical Imaging (pp. 93-100). Springer, Cham.

[31] Nyúl, L.G., Udupa, J.K. and Zhang, X., 2000. New variants of a method of MRI scale standardization. IEEE transactions on medical imaging, 19(2), pp.143150 .

[32] Glocker, B., Zikic, D., Konukoglu, E., Haynor, D.R. and Criminisi, A., 2013, September. Vertebrae localization in pathological spine CT via dense classification from sparse annotations. In International Conference on Medical Image Computing and Computer-Assisted Intervention (pp. 262-270). Springer, Berlin, Heidelberg.

[33] Criminisi, A., Robertson, D., Konukoglu, E., Shotton, J., Pathak, S., White, S. and Siddiqui, K., 2013. Regression forests for efficient anatomy detection and localization in computed tomography scans. Medical image analy-sis, 17(8), pp.1293-1303.

[34] Schmid, J. and Magnenat-Thalmann, N., 2008, September. MRI bone segmentation using deformable models and shape priors. In International conference on medical image computing and computer-assisted intervention (pp. 119-126). Springer, Berlin, Heidelberg.

[35] Volino, P., \& Magnenat-Thalmann, N. (2000). Implementing fast cloth simulation with collision response. In Computer Graphics International, 2000. Proceedings (pp. 257-266). IEEE.

[36] Cootes, T.F., Hill, A., Taylor, C.J. and Haslam, J., 1993, June. The use of active shape models for locating structures in medical images. In Biennial International Conference on Information Processing in Medical Imaging (pp. 33-47). Springer, Berlin, Heidelberg.

[37] Kraevoy, V. and Sheffer, A., 2006. Mean-value geometry encoding. International Journal of Shape Modeling, 12(01), pp.29-46.

[38] Kumar, S., 2003, October. Discriminative random fields: A discriminative framework for contextual interaction in classification. In Computer Vision, 2003. Proceedings. Ninth IEEE International Conference on (pp. 1150-1157). IEEE. 
[39] Chu, C., Chen, C., Liu, L. and Zheng, G., 2015. Facts: fully automatic ct segmentation of a hip joint. Annals of biomedical engineering, 43(5), pp.12471259 .

[40] Yushkevich, P.A., Piven, J., Hazlett, H.C., Smith, R.G., Ho, S., Gee, J.C. and Gerig, G., 2006. User-guided 3D active contour segmentation of anatomical structures: significantly improved efficiency and reliability. Neuroimage, 31(3), pp.1116-1128.

[41] Fedorov, A., Beichel, R., Kalpathy-Cramer, J., Finet, J., Fillion-Robin, J.C., Pujol, S., Bauer, C., Jennings, D., Fennessy, F., Sonka, M. and Buatti, J., 2012. 3D Slicer as an image computing platform for the Quantitative Imaging Network. Magnetic resonance imaging, 30(9), pp.1323-1341.

[42] Zikic, D., Glocker, B., Konukoglu, E., Criminisi, A., Demiralp, C., Shotton, J., Thomas, O.M., Das, T., Jena, R. and Price, S.J., 2012, October. Decision forests for tissue-specific segmentation of high-grade gliomas in multi-channel MR. In International Conference on Medical Image Computing and Computer-Assisted Intervention (pp. 369-376). Springer, Berlin, Heidelberg.

[43] Mahapatra, D., 2014. Analyzing training information from random forests for improved image segmentation. IEEE Transactions on Image Processing, 23(4), pp.1504-1512.

[44] Montillo, A., Shotton, J., Winn, J., Iglesias, J.E., Metaxas, D. and Criminisi, A., 2011, July. Entangled decision forests and their application for semantic segmentation of CT images. In Biennial International Conference on Information Processing in Medical Imaging (pp. 184-196). Springer, Berlin, Heidelberg.

[45] Zikic, D., Glocker, B. and Criminisi, A., 2014. Encoding atlases by randomized classification forests for efficient multi-atlas label propagation. Medical image analysis, 18(8), pp.1262-1273.

[46] Geremia, E., Clatz, O., Menze, B.H., Konukoglu, E., Criminisi, A. and Ayache, N., 2011. Spatial decision forests for MS lesion segmentation in multichannel magnetic resonance images. NeuroImage, 57(2), pp.378-390.

[47] Louppe, G., 2014. Understanding random forests: From theory to practice. arXiv preprint arXiv:1407.7502.

[48] Criminisi, A. and Shotton, J. eds., 2013. Decision forests for computer vision and medical image analysis. Springer Science \& Business Media.

[49] Damopoulos, D., Glocker, B. and Zheng, G., 2017, September. Automatic localization of the lumbar vertebral landmarks in CT images with context features. In International Workshop and Challenge on Computational Methods and Clinical Applications in Musculoskeletal Imaging (pp. 59-71). Springer, Cham.

[50] Breiman, L., 2001. Random forests. Machine learning, 45(1), pp.5-32.

[51] S. Kosov, Direct Graphical Models C++ Library, 2013, (http://research.project-10.de/dgm/).

[52] Woolson, R.F., 2007. Wilcoxon signed-rank test. Wiley encyclopedia of clinical trials, pp.1-3.

[53] Li, H. and Johnson, T., 2014. Wilcoxon's signed-rank statistic: what null hypothesis and why it matters. Pharmaceutical statistics, 13(5), pp.281-285. 
[54] Sheskin, D.J., 2003. Handbook of parametric and nonparametric statistical procedures. cre Press, pp.158-165. 


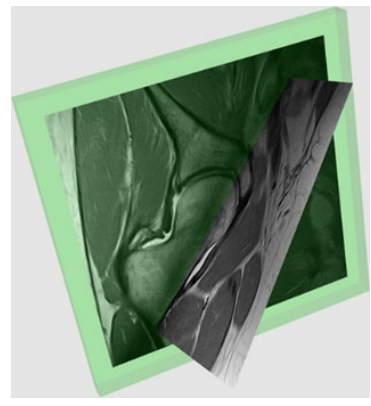

(a)

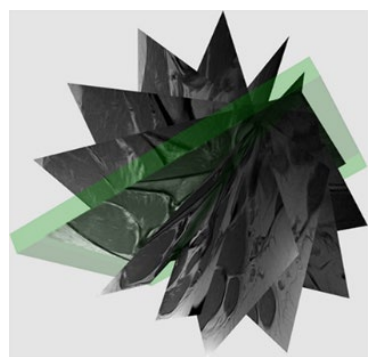

(c)

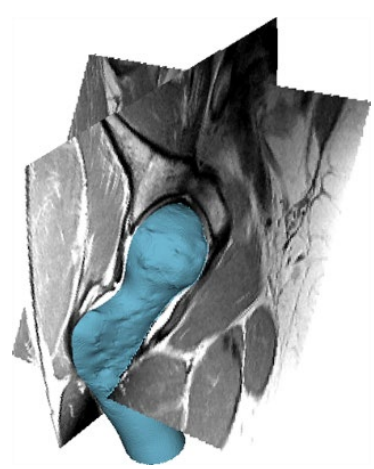

(e)

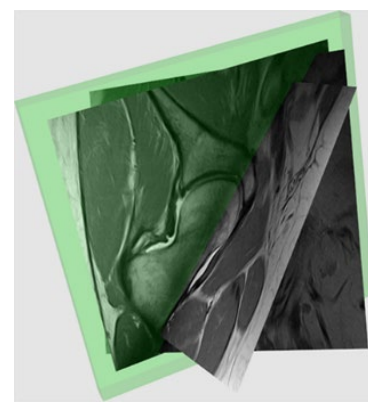

(b)

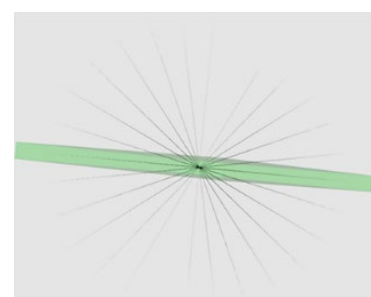

(d)

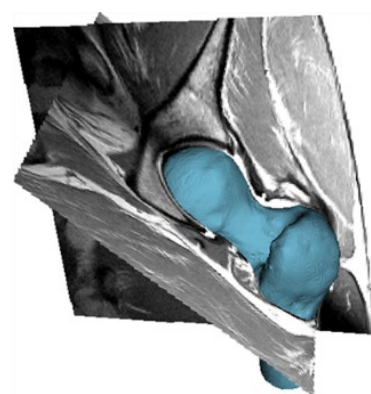

(f)

Fig. 1. Visualization of the 3D geometry of a radial scan along with the registered 3D model, shown from different 3D viewpoints. In (a)-(d), the same image is highlighted with a green overlay. For clarity of visualization, only two, three and seven of the total 14 images of the radial scan are shown in (a), (b) and (c) respectively. In (d), it is can be observed that all the images share a common axis and that their angular displacements around their common axis are uniformly distributed around the circle. Finally, in (e) and (f), the two images of (a) are shown along with the registered 3D model, as generated by the proposed method. Subfigures (e) and (f) were created using the 3D Slicer software [41]. 


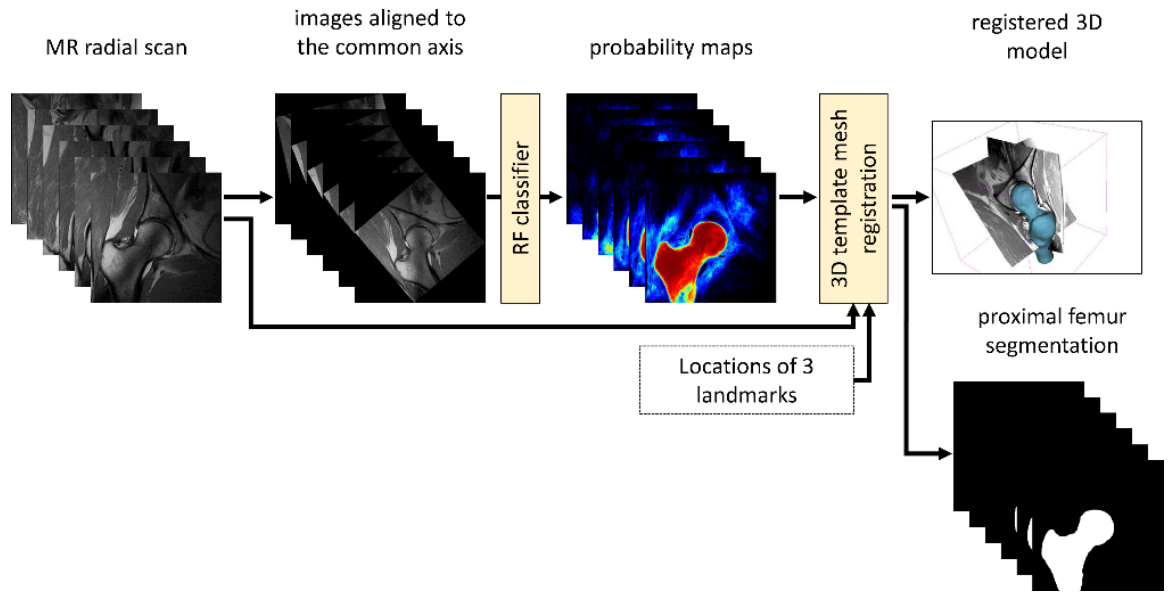

Fig. 2. Summary of the pipeline. (a) The images of a radial scan are preprocessed and they are aligned according the common axis of the scan; (b) The random forest classifier makes a prediction for every pixel of the images on whether in corresponds to femur. The generated probability maps are rotated back to the original orientations; (c) a 3D template mesh of the femur is registered to the radial images using the generated probability maps to drive the registration process and the positions of the three landmarks to initialize it. 


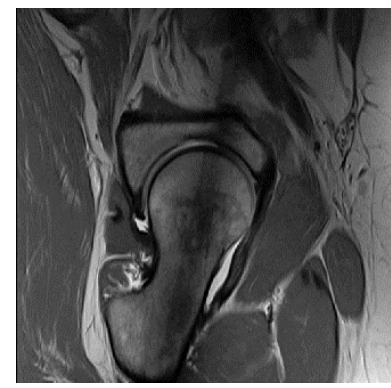

(a)

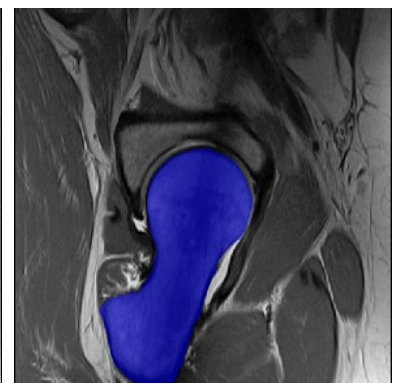

(b)

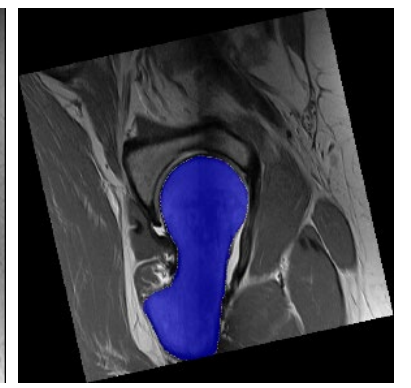

(c)

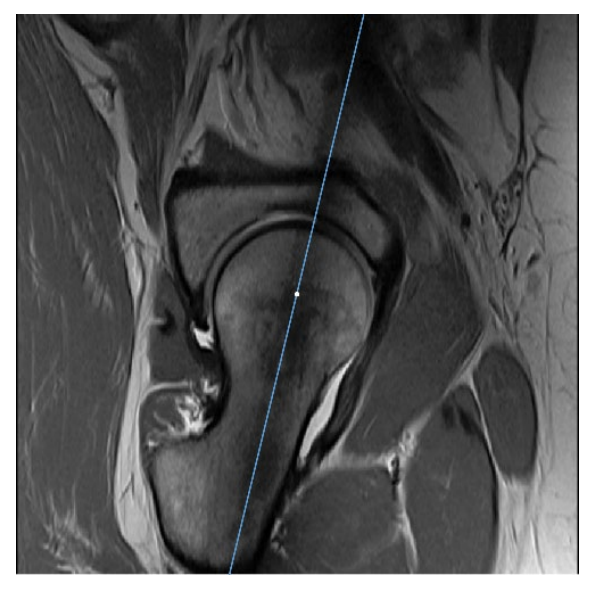

(d)

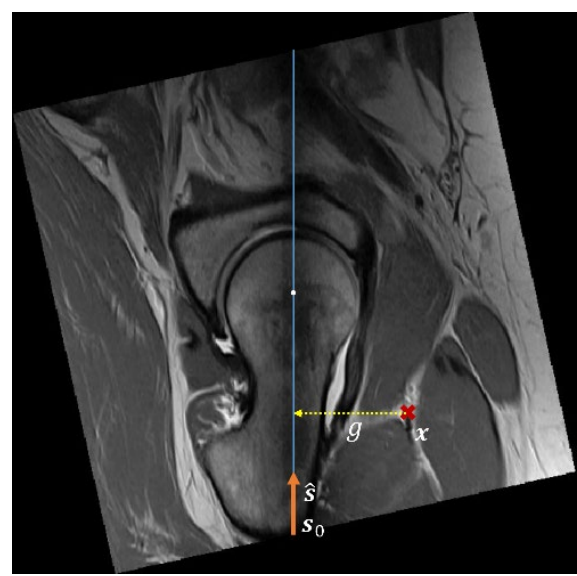

(e)

Fig. 3. Alignment of the images with respect to the common axis and the geometric. From (a) to (c): An example input image; the same image, with the ground truth segmentation of the femur highlighted with a blue overlay; the appearance of the image, when it is aligned with respect to the common axis (the image has been rescaled on this figure to make the figure clearer). The crosstalk artifact, occurring on the common axis of all the images and typical for MR radial scans, can be easily observed. (d): The image of subfigure (a), with the common axis pinpointed with a blue line. (e) The aligned image of subfigure (c), with the common axis pinpointed with a blue line. The unit vector $\hat{\boldsymbol{s}}$ is the direction vector of the common axis. Since the image is aligned, $\hat{\boldsymbol{s}}$ is parallel to the vertical axis. The length of the yellow dotted line is the value of the geometric feature $g$, computed at the point $\boldsymbol{x}$. 

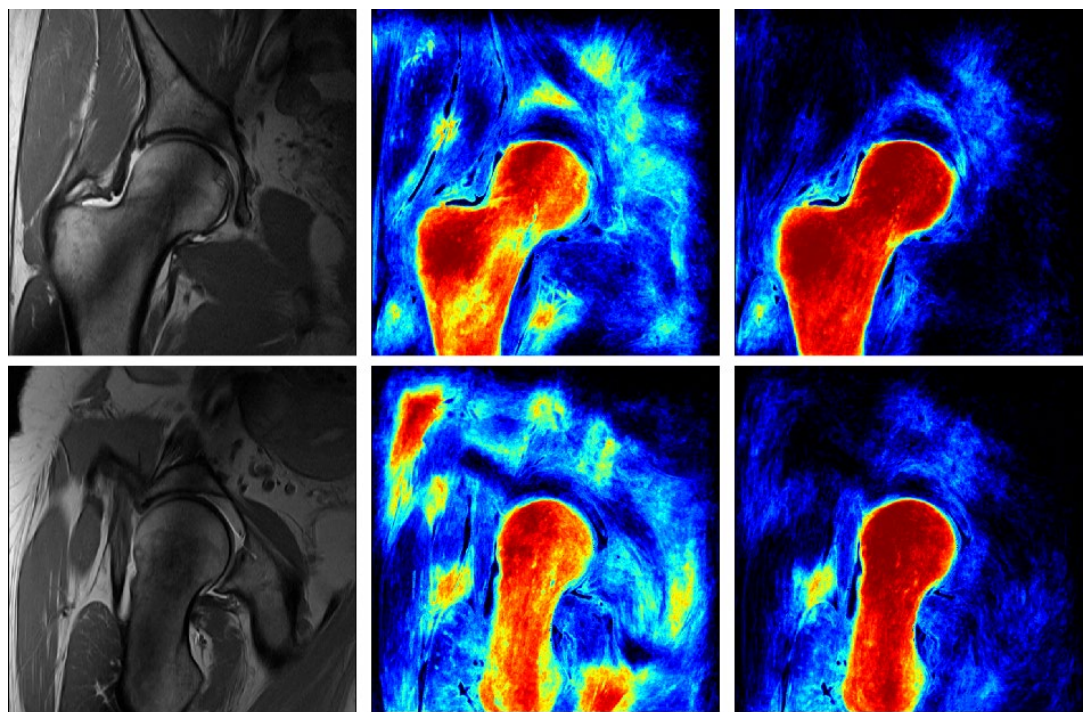

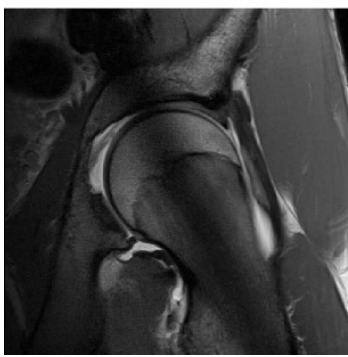

(a)

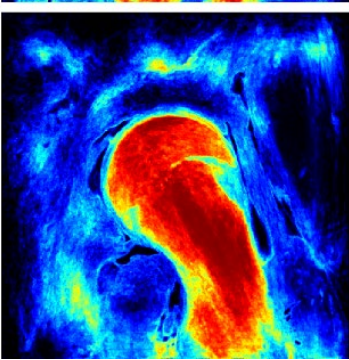

(b)

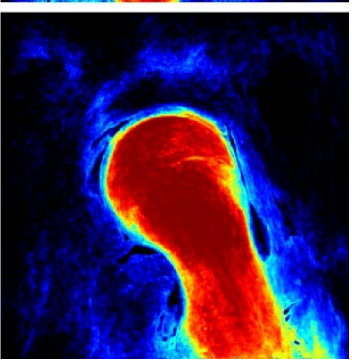

(c)

Fig. 4. Probability maps with and without using the geometric feature. (a) three testing images; (b) The probability maps generated by a classifier that used only the intensity-based features; (c) The probability maps generated by a classifier that used both the intensity-based and the geometric feature. The probability maps were visualized using the ITK-Snap software [40]. 


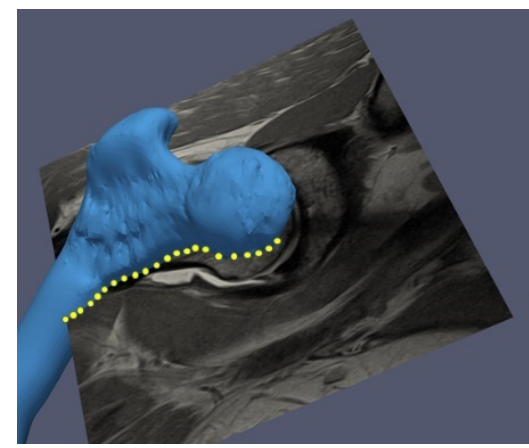

(a)

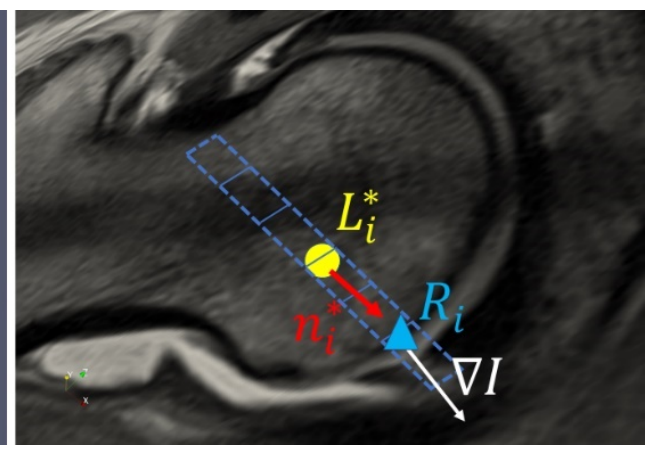

(b)

Fig. 5. Search of target point $R_{i}$ for the image force. (a) Mesh vertices close enough to the image plane and whose projection $L_{i}^{*}$ is within image bounds are selected (yellow $\bullet$ ). (b) For each vertex projection $L_{i}^{*}(\bullet)$, values are sampled along the projected normal direction $n_{i}^{*}$ at regular steps $s$. The sampled position at which the image gradient $\nabla \mathrm{I}$ is the largest and the best aligned with $n_{i}^{*}$ is chosen as the target point $R_{i}(\mathbf{\Delta})$. 

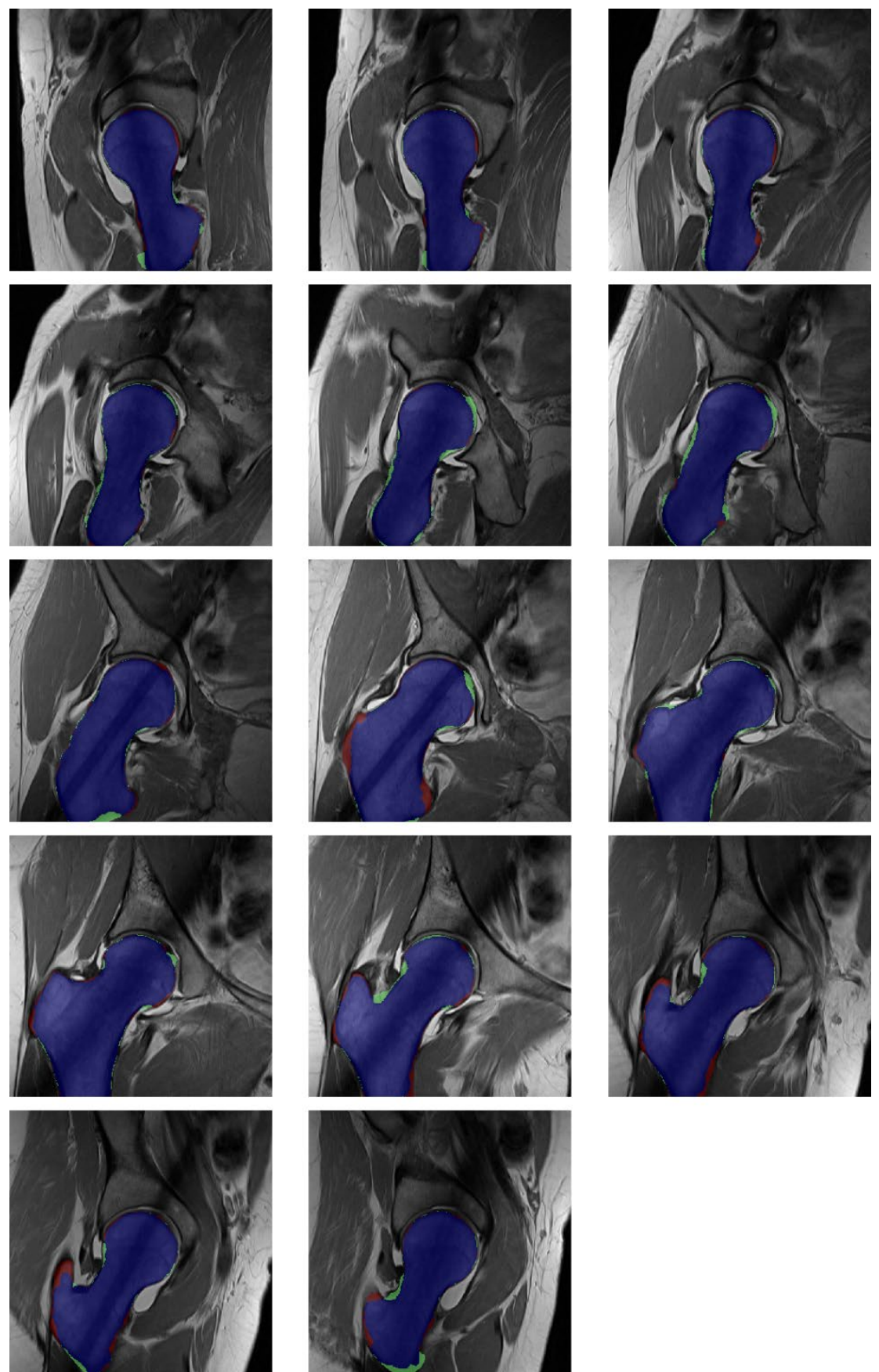

Fig. 6. Segmentation result for the 14 images of the case with the median DSC (96.65\%) and illustration of its errors with respect to the manual, reference segmentation. Blue: true positive pixels; red: false negative pixels; green: false positsive pixels. 


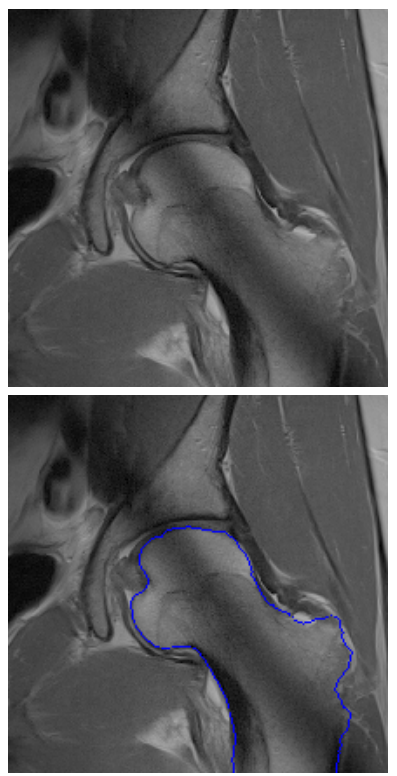

(a)
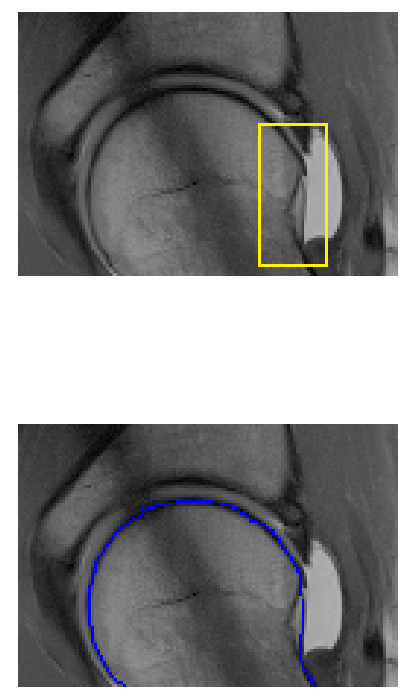

(d)

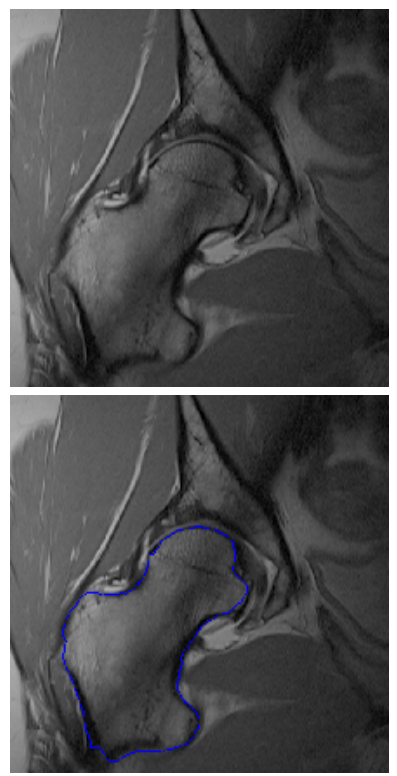

(b)
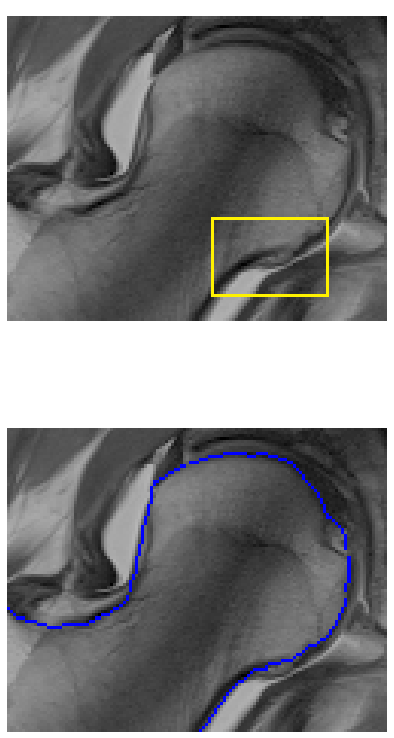

(e)

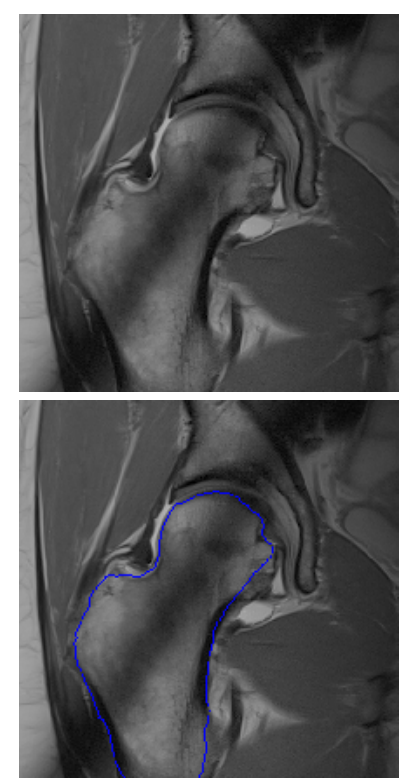

(c)
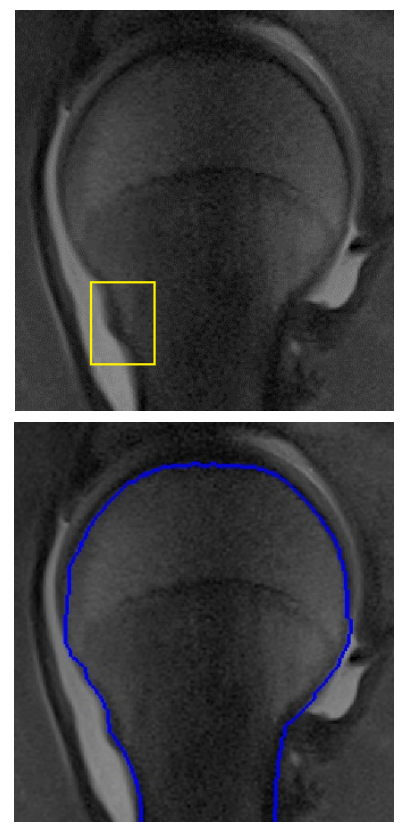

(f) 
Fig 7. Visualization of six representative radial images with pathological findings and/or presenting challenges for the segmentation, each corresponding to a different patient. (a) - (c): Nonspherical shape of femur head; (d) - (f): lesions of cam-type FAI (femoral "bumps"). Below every image, the border of the segmentation achieved by the pipeline is also shown. In (c) and (e), the result of the pipeline has visible mistakes. In (c), the border of a sub-region of the femoral head is not captured correctly and in (e) a lesion is segmented out. 

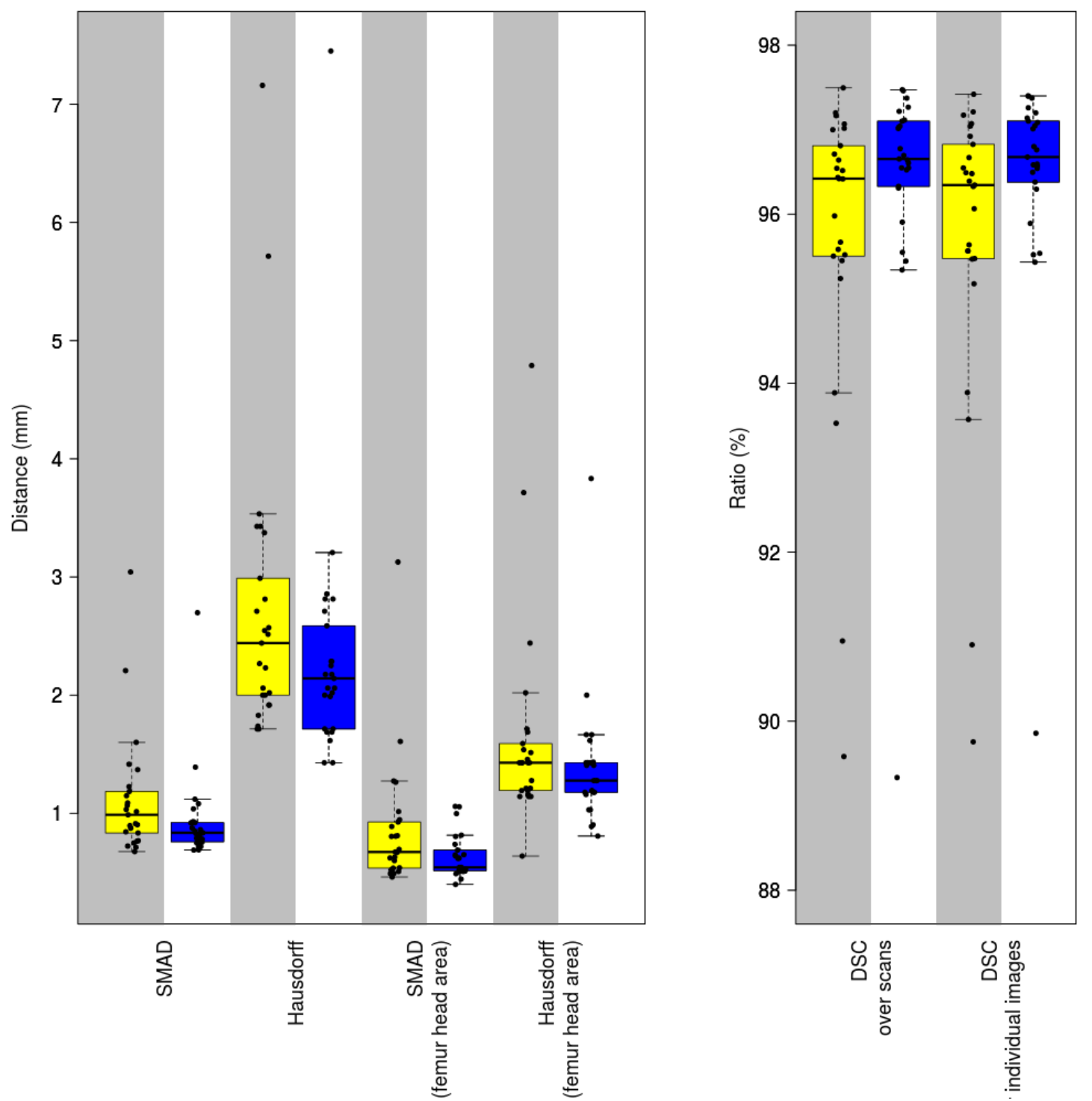

Fig 8. Box-and-whisker plots of the proposed pipeline and the its purely classifier-based variant for all the six evaluation metrics. The proposed pipeline corresponds to the blue boxes (gray background) and the purely classifier-based variant corresponds to the yellow boxes (white background). For clarity of visualization, the datapoints have been jittered horizontally. The boxes span from the lower quartile $\left(\mathrm{Q}_{1}\right)$ to the higher quartile $\left(\mathrm{Q}_{3}\right)$ and the band within them corresponds to the median value. Thus, the span of the boxes is equal to the IQR. The two whiskers lay on lowest datapoint still within 1.5 IQR of the lower quartile and on the highest datapoint still within 1.5 IQR of the upper quartile. 
Table 1. Performance of the proposed pipeline. The DSC over whole scans (each one consisting of 14 images) and the DSC over individual images are both presented. The symmetric mean absolute distance (SMAD) and the Hausdorff distance are computed for both the whole proximal femur and for the femoral head area only.

\begin{tabular}{|l|r|r|r|r|r|}
\hline & \multicolumn{1}{|c|}{ Mean } & Std. Dev. & \multicolumn{1}{c|}{ Median } & \multicolumn{1}{l|}{ Min. } & \multicolumn{1}{c|}{ Max. } \\
\hline DSC over scans (\%) & 96.37 & 1.55 & 96.65 & 90.90 & 97.47 \\
\hline DCS over images (\%) & 96.40 & 1.45 & 96.68 & 89.86 & 97.40 \\
\hline SMAD (mm) & 0.94 & 0.39 & 0.84 & 0.69 & 2.20 \\
\hline $\begin{array}{l}\text { SMAD, only femoral } \\
\text { head (mm) }\end{array}$ & 0.64 & 0.18 & 0.54 & 0.40 & 1.06 \\
\hline Hausdorff (mm) & 2.37 & 1.13 & 2.14 & 1.43 & 4.35 \\
\hline $\begin{array}{l}\text { Hausdorff, only fem- } \\
\text { oral head (mm) }\end{array}$ & 1.41 & 0.56 & 1.28 & 0.81 & 3.83 \\
\hline
\end{tabular}


Table 2. Performance of a purely classifier-based pipeline, without the registration of a 3D model. A Wilcoxon signed-rank test was performed for each of the six evaluation metrics in order to determine whether the differences with the full pipeline (Table 1) are statistically significant. After the conduction of the six tests the Holm-Bonferroni correction method was applied and the resulting (corrected) p-values are listed in the rightmost column.

\begin{tabular}{|l|r|r|r|r|r|r|}
\hline & Mean & \multicolumn{1}{|l}{$\begin{array}{l}\text { Std. } \\
\text { Dev. }\end{array}$} & Median & \multicolumn{1}{l|}{ Min. } & Max. & p-value \\
\hline $\begin{array}{l}\text { DSC over } \\
\text { scans (\%) }\end{array}$ & 95.69 & 1.87 & 96.42 & 89.58 & 97.49 & 0.0100 \\
\hline $\begin{array}{l}\text { DSC over in- } \\
\text { dividual im- } \\
\text { ages (\%) }\end{array}$ & 95.68 & 1.84 & 96.35 & 89.75 & 97.42 & 0.0046 \\
\hline SMAD (mm) & 1.12 & 0.51 & 0.99 & 0.68 & 3.04 & 0.0103 \\
\hline $\begin{array}{l}\text { SMAD, only } \\
\text { femoral head } \\
\text { (mm) }\end{array}$ & 0.86 & 0.54 & 0.67 & 0.46 & 3.13 & 0.0001 \\
\hline $\begin{array}{l}\text { Hausdorff } \\
\text { (mm) }\end{array}$ & 2.75 & 1.24 & 2.44 & 1.71 & 7.16 & 0.0488 \\
\hline $\begin{array}{l}\text { Hausdorff, } \\
\text { only femoral } \\
\text { head (mm) }\end{array}$ & 1.64 & 0.85 & 1.43 & 0.64 & 4.79 & 0.0488 \\
\hline
\end{tabular}


Table 3. Performance when the geometric feature is not used. As in Table 2, a Wilcoxon signedrank test was performed for each of the six evaluation metrics to determine whether the differences with the full pipeline (Table 1) are statistically significant. After the conduction of the six tests the Holm-Bonferroni correction method was applied and the resulting (corrected) p-values are listed in the rightmost column.

\begin{tabular}{|l|r|r|r|r|r|r|}
\hline & Mean & \multicolumn{1}{|c|}{$\begin{array}{l}\text { Std. } \\
\text { Dev. }\end{array}$} & Median & Min. & Max. & p-value \\
\hline $\begin{array}{l}\text { DSC over } \\
\text { scans (\%) }\end{array}$ & 95.80 & 2.32 & 96.40 & 85.04 & 97.39 & 0.0007 \\
\hline $\begin{array}{l}\text { DSC over in- } \\
\text { dividual im- } \\
\text { ages (\%) }\end{array}$ & 95.78 & 2.36 & 96.35 & 85.29 & 97.33 & 0.0008 \\
\hline SMAD (mm) & 1.08 & 0.53 & 0.98 & 0.70 & 3.48 & 0.0013 \\
\hline $\begin{array}{l}\text { SMAD, only } \\
\text { femoral head } \\
\text { (mm) }\end{array}$ & 0.74 & 0.33 & 0.66 & 0.43 & 2.07 & 0.0125 \\
\hline $\begin{array}{l}\text { Hausdorff } \\
\text { (mm) }\end{array}$ & 2.59 & 1.42 & 2.25 & 1.44 & 9.08 & 0.1122 \\
\hline $\begin{array}{l}\text { Hausdorff, } \\
\text { only femoral } \\
\text { head (mm) }\end{array}$ & 1.61 & 0.71 & 1.46 & 1.14 & 4.87 & 0.0170 \\
\hline
\end{tabular}

